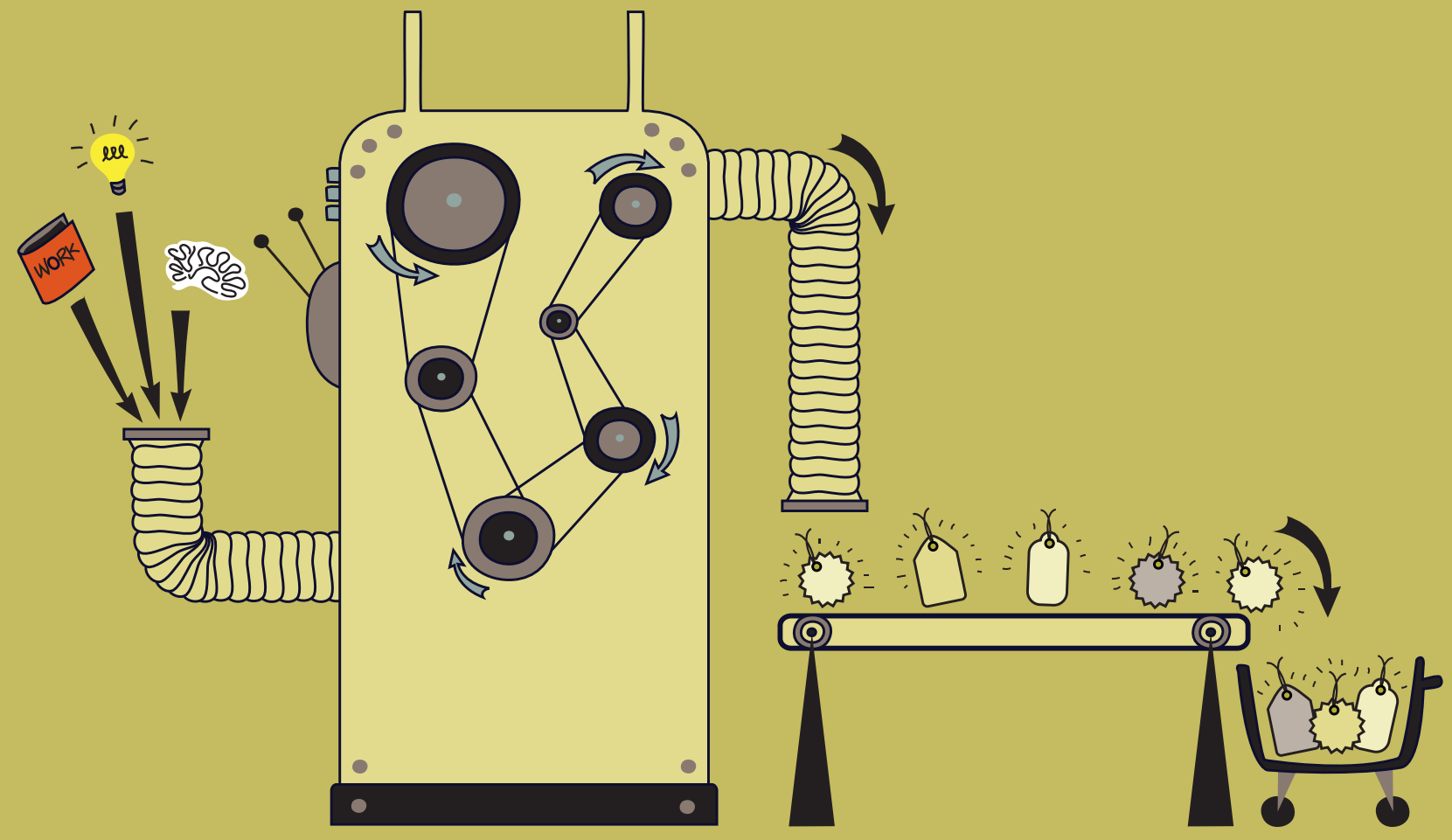




\title{
DYNAMIC PRICING SUPPORT SYSTEMS FOR DIY RETAILERS - A CASE STUDY FROM AUSTRIA
}

\author{
Martin Natter, Thomas Reutterer and Andreas Mild
}

\begin{abstract}
Merchandise managers have long dreamt of automated dynamic systems to help them make well-informed pricing decisions. However, such systems have proved as elusive as the Holy Grail - until now, that is. The story of an Austrian DIY retailer shows often undetected opportunities to use valuable information, hidden in retailers' data warehouses, on consumer reactions to previous price changes in order to make automatic pricing and promotion decisions.
\end{abstract}

Effective pricing has always been something of a challenge for retailers with extensive product ranges. Managing the pricing of a wide variety of items, and responding effectively to changes in supply and demand, is a highly complex, labour-intensive and time-consuming task. Add to this the demands of promotions and the impact of competitor pricing, and it is not hard to see why simple solutions, such as high-low pricing strategies and the rationalization of the product range, have long found favor.

An Austrian DIY retailer, bauMax, with over 120 stores, sales of approximately $€ 1.25$ billion in nine central and eastern European countries, and a 25 percent market share in its domestic market, appears to have achieved the Holy Grail of retailing, long thought to be near-impossible.

It has successfully developed an automated dynamic pricing system, designed to support retail purchasing, merchandising and marketing managers in their pricing and promotion decisions. The system automatically pro- cesses article-specific pricing and promotion recommendations, ensures no article is out of stock, accounts for indirect contributions from lift effects, and is applicable across regions and countries, taking into account the differences in taxation and currencies.

Clearly, the development of such a system requires significant investment, but bauMax shows the rewards can be great. Once up and running, it produced a 2 percent increase in sales and an 8 percent rise in gross profits.

\section{Developing an accurate model of pricing behavior}

The heart of bauMax's system is a weekly demand model for 60,000 stock-keeping units, incorporating a wide range of factors that impact pricing, such as seasonality, item availability, discounts, and other reference effects.

The challenge for any company thinking about developing such a system is how to accurately capture customers' purchasing behavior without producing a model that is overly complex. Building simple but accurate models is of crucial importance to retailers selling so many different products.

bauMax's model uses a unique combination of equations that aims to reflect the relationship between price and demand in the real world. These capture not only how customers respond to changes in the price of a single item, but also the influence of simple external factors such as seasonal demand fluctuations, and more complex ones such as complementary or substitutional effects (Refer to box, next page).

\section{THE AUTHORS}

Martin Natter, Hans Strothoff Professor of Retail Marketing at the Johann Wolfgang Goethe-University Frankfurt, Germany, natter@wiwi.uni-frankfurt.de

Thomas Reutterer,

Associate Professor at the Institute for Retailing and Marketing, Vienna University of Economics and Business Administration, Austria, thomas.reutterer@wu-wien.ac.at

Andreas Mild,

Associate Professor at the Institute for Production Management, Vienna University of Economics and Business Administration, Austria,

andreas.mild@wu-wien.ac.at

The article is based with the permission of INFORMS on Natter, M., Reutterer, T., Mild, A. and Taudes, A. (2007): An Assortment-Wide DecisionSupport System for Dynamic Pricing and Promotion Planning in DIY Retailing, Marketing Science, Vol. 26 (4), 576-583. 
$\{B o x\}$

\section{THE HEART OF THE PRICING MACHINE}

The bauMax pricing decision-support model uses six core equations to express the relationships between price, demand, and profit. A seventh equation was added at a later date (for details, see the main text).

\section{Equation 1}

Individual transaction histories show that the average DIY shopper is highly price conscious. The system represents this in a form known technically as an 'unobserved reference price', modeled as an exponentially smoothed function. Customers frequently make a mental comparison of prices not only at the time of purchase, but when checking the bill at home, putting an item away, or even when using it for the first time. Inherent in the model's equation is the assumption that the store's price-sensitive customers adapt their expectations more rapidly to price reductions than to price increases. This assumption is supported by the fact that stores advertise reductions rather than increases.

\section{Equation 2}

The second equation establishes the impact of pricing and availability on stock levels. In addition to information on the price and reference price of an item, it takes into account the impact of current demand trends, seasonality, the number of branches, discounts, and so on. Seasonality plays an important role in shaping demand in categories such as gardening tools and building materials. Differences in the stock levels of the various outlets can have an impact on demand at the store level. The model also makes it explicit that overly frequent price promotions will cause the price to drift towards the lower end of the range.

\section{Equation 3}

This takes account of asymmetric interdependencies in pricing. It does so by calculating the item-specific profit-lift effects related to direct and indirect discounts, based on shopping basket data.

\section{Equation 4}

This tests for complementary cross-effects. The equation is based on the item-level calculation of two interrelated probabilities: that of choosing item $x$ when item $y$ is already in the basket, and that of observing item $x$ in baskets that do not contain item $y$.

\section{Equation 5}

This takes into account the fact that substitution effects are only observed in about 10 percent of bauMax's product range. It also reflects the conditional profit contribution (either positive or negative) of interdependencies producing product lift.

\section{Equation 6}

This adds substitution effects into the scenario. As the retailer is only concerned with the impact of substitution on profits, the equation multiplies the lift effect by the difference in the profit contribution.
Natter, M., Reutterer, T., Mild, A. and Taudes, A. (2007): An Assortment-Wide Decision Support System for Dynamic Pricing and Promotion Planning in DIY Retailing. Marketing Science, Vol. 26 (4), 576-583. 
Building a smart but manageable revenue management system is only one part of the story. The building blocks of the bauMax dynamic pricing system show that the data and computational issues involved in such a project are hugely challenging. (Refer to Figure 1).

The data needs to be routinely retrieved from such diverse sources as inventory management, marketing information and point-of-sale scanning systems. In order to estimate the model parameters, the data must be refined and interlinked. Fortunately, bauMax had already implemented an advanced company-wide data warehouse solution, which facilitated many of these data pre-processing operations.

The computational burden of repeatedly evaluating several thousand item-specific market response models and then determining optimal prices shows the importance of keeping the demand models as simple as possible. In addition, the huge product range and significant computational restrictions made it unfeasible to account for all possible cross-item purchase correlations in the model. bauMax's pricing support system resolves this issue by incorporating a proxy for the complex interrelationship structure of complementary and substitutional purchase effects in the item-level profit functions to be optimized by the system. This is accomplished by the so-called profit-lift effect measure, which is determined by mining bauMax's shopping basket data for significant deviations from expected item-specific purchase inter-correlations (equations 3-5).

However clever and sophisticated bauMax's system might be, there is a world of difference between possessing a shiny new tool and ensuring that it is used in a proper and effective manner. The challenge for bauMax was to ensure that the dynamic pricing system was positioned within the organization in a manner that would ensure that its pricing recommendations were acted upon.

To address this challenge, bauMax located the system within a three-step process that starts with managerial input and ends with performance monitoring.

In the initial step, managerial input is required to establish the underlying pricing conditions. This is also the point at which any necessary modifications, based on the feedback and experience from the previous rounds of pricing, are incorporated into the system.

The senior managers will need to answer a number of critical questions to set the boundary conditions for the system. For instance, are there any current limits to marketing resources? Will the existing business conditions impose any new constraints on prices? The system claims neither to be a substitute for managerial experience in answering such questions, nor to be fully comprehensive. One of its present constraints, for instance (though this may well be addressed at a later stage of development), is that the system makes little allowance for price differences across the various sales channels of the company. (Refer to Figure 2, next page)

Once these decisions have been made and the feedback from previous rounds has been incorporated, the automated system takes over. It retrieves the necessary data from the data warehouse and then generates the optimal prices for each item within the retailer's offer. These decisions are transferred to store managers automatically, thereby ensuring that up-to-minute information is available at the point of sale.

This is not the end of the story, however. No merchandise manager responsible for price and promotion planning at bauMax likes to be overridden by an automated system, so the process provides room for managers to reject or modify its pricing recommendations. If they do so, however, they must record the reasons for their decision.

This step has proved highly useful in improving the effectiveness of the model from a number of perspectives. Initially, it did not take into account the merchandise managers' preference for maintaining the uniformity of product families (for example in terms of different color
FIGURE 1

A typical bauMax store layout 
Figure 2:

PRICING PROCESS

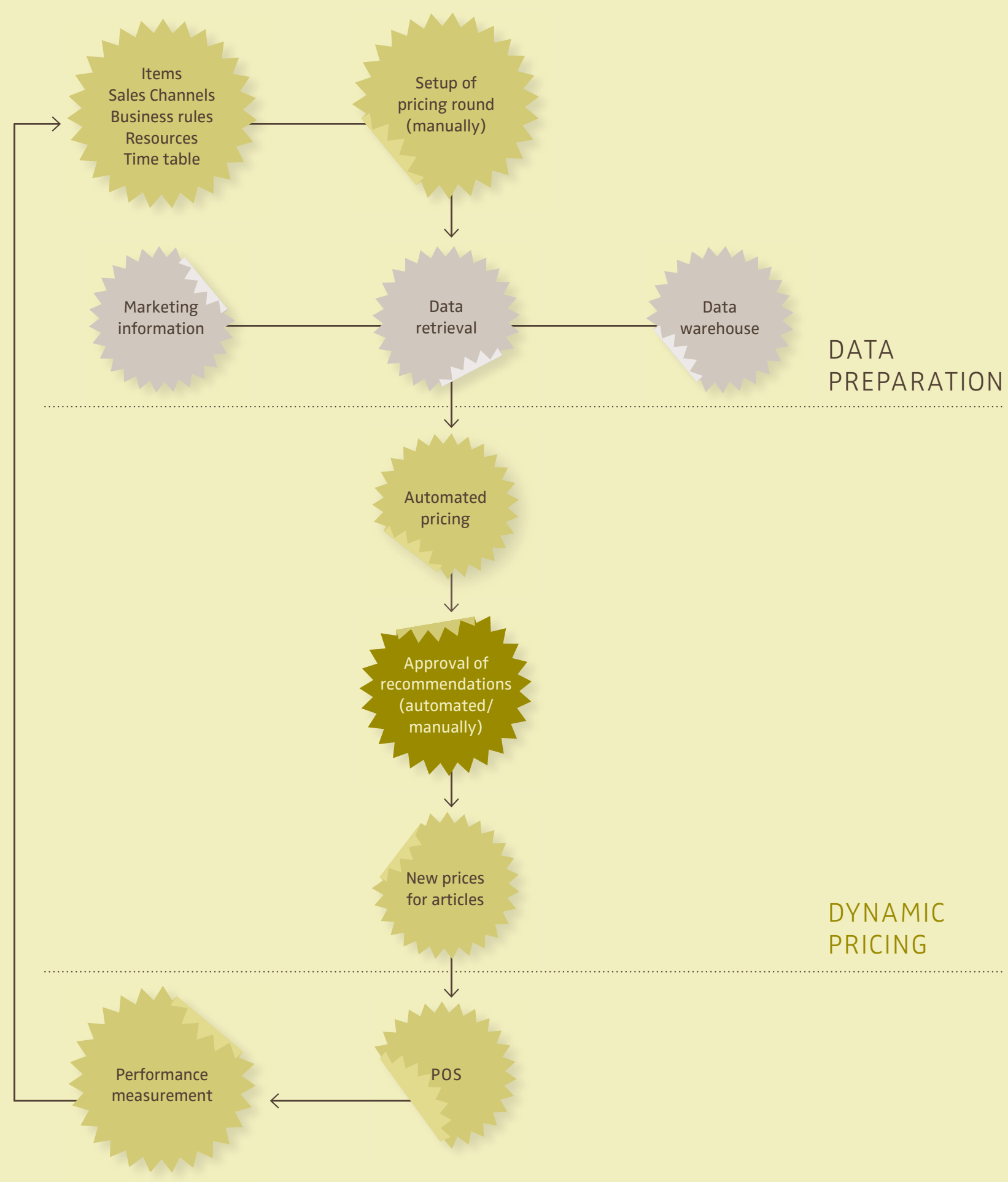


variants or package sizes). The discrepancies generated by the system, for example introducing price differences between colors, quickly proved unacceptable to the merchandise managers. However, once they had a chance to make their objections known through the feedback process, these concerns could be responded to and the model adjusted as appropriate.

A second area of concern for the managers was the system's recommendations to raise prices in circumstances where they knew competitors' prices to be lower. Initially, they regularly overruled these decisions. However, once the reasons for this pattern were understood through the feedback process, the system was modified, providing a better explanation of why price rises had been recommended. This information might include, for instance, the fact that demand for a particular item was known to be relatively inelastic, or that customers showed a low level of price consciousness regarding an item. This was an important lesson for the team implementing the system. The extra information they added helped to reassure and convince the store managers, raising their overall acceptance of the system's recommendations.

The final step monitors and evaluates the effectiveness of all pricing decisions, including those in which the store manager has intervened, in terms of their effect on profits and sales. This information is then fed back to the senior managers and action taken, wherever necessary, to fine-tune the model. This feedback loop ensures that the model goes through a process of continuous improvement.

Fully involving merchandising managers

in the implementation process

Introducing any new tool into the decision-making processes of a large retail business is always something of a challenge. The usual approach is to ensure that the tool is first piloted and then rolled out across the organisation. However, a system as complex as bauMax's presents a challenge of a higher order. Not only does pricing lie at the heart of retail profitability, making the risks involved in introducing such a tool significant, but the tool itself will need to be proved on a wide range of items, and in a variety of circumstances, if the trials are to be of any real worth. The trials also need to take account of the likely response of users. If the pricing tool were to be rolled out too quickly, for instance, before all glitches had been properly ironed out, then resistance to its use could build up among the merchandise managers who are its key users.

\section{») Introducing any new tool into the decision-making processes of a large retail business is always some- thing of a challenge. «}

No one at bauMax wanted to see the worst-case scenario, in which the trials went badly wrong and endangered the retailer's profitability. It resolved these challenges by introducing a three-phase implementation program that incorporated room for modification in each phase.

In the first, the system was used to price a limited number of items in just ten representative stores in Austria. To ensure the system was behaving as expected, the performance of these items was benchmarked against a set of control stores with similar characteristics to those in which the system was being piloted.

This phase turned out to be extremely important in convincing the initially rather skeptical managers of the new system's effectiveness. For the approximately 1,900 articles involved in the initial pricing round, profit differences between test and reference outlets were computed and cumulated over a period of weeks. When relative profits increased by 3.6 percent only six weeks after starting the first pricing round, management rolled out the new prices to all Austrian outlets by week 41 . After this, profits also increased in the remaining outlets and differences diminished over the next few weeks until the second price round began. The following price rounds showed similar patterns, which significantly increased managers' acceptance of using the system on a wider scale. (Refer to figure 3, next page)

In the first few rounds, only the core system equations were tested. In subsequent rounds, the number of items was gradually increased and the profit-lift functions incorporated into the system trial. 
Figure 3:

CUMULATED PROFIT DIFFERENCES (IN PERCENT)

BETWEEN TEST AND REFERENCE OUTLETS

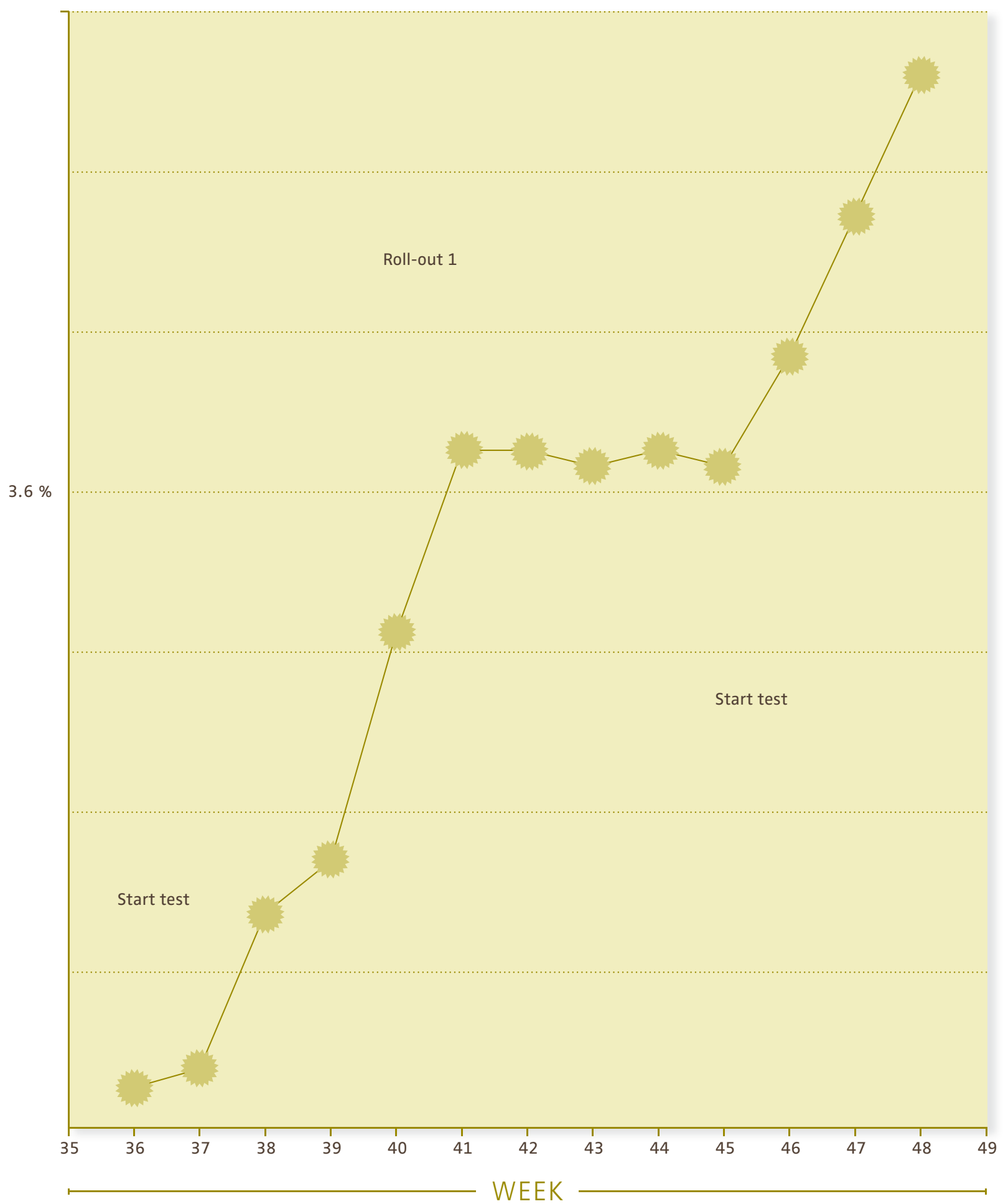


In phase two, bauMax took stock of the results of phase one and made a number of modifications to the system. It also extended the range of stores taking part in the trial, including outlets outside Austria for the first time.

The most significant changes made during this phase resulted from the fact that phase one showed a need to take account of the pricing system's impact on sales volumes. Following the introduction of the system, and although the profitability of each tracked item increased as predicted, higher prices sometimes led to decreased sales. The merchandise managers were therefore understandably worried that improved profitability might cause them to miss their sales and volume targets.

Following discussions with the managers, bauMax added equation 7 to the decision support model. This added an array of commonly used pricing strategies and gave an explicit weighting to each of them that reflects their relative impact on the resulting gross profits. Although the overall pricing system continues to reflect the owner's and CFO's emphasis on maximizing profitability, it now also takes into account the relationship between price and demand, and its impact on sales volumes.

This new equation not only improved the effectiveness of the decision support system, but was also a very significant factor in raising its level of acceptance among the merchandising managers. On the one hand, the process of introducing these modifications had given them the chance to get to grips with how the system actually worked. On the other, the managers were now sure that it reflected their own merchandising experience.

Phase three started eighteen months after the project began, and rolled out the system to all the stores across the company. In this phase, and subsequently, the pricing rounds were carried out twice monthly. Each round incorporates both management's recommendations in line with the company strategy, and feedback from store managers.

\section{Implications of automated pricing \\ systems for retailing}

The introduction of bauMax's system has not only led to increased sales, but has also had a very positive impact on profits. With such results, and in today's cashconstrained times, the question on every executive's lips is therefore likely to be whether a similar system would be applicable in other contexts.
The experience indicates that automated pricing systems can potentially have a similar impact in businesses and industries which fulfil three specific criteria:

$>$ They trade predominantly in fast-moving consumer goods (slow-moving items would require some modifications of this model)

> They have medium- or long-term product cycles (this excludes fashion and electronics products, for instance, which tend to have short cycles)

> They have access to sufficient historical data on price changes. bauMax did not, and had seen very limited price movement in a large number of items, but overcame this problem by carrying out a series of experiments to develop a database of price elasticity and price effects.

Though the impact of this system has already been substantial, bauMax has its eye on a series of further developments. As yet, the system has only a limited ability to deal with competitor data - it has started by tracking the prices of the 100 leading articles on which competitors focus. Future developments are likely to extend this range. One particularly interesting research area is the creation of an integrated demand-andsupply-chain management model that will link pricing to inventory and production planning.

There are a number of information technology improvements that can be expected to further enhance marketing intelligence solutions like that implemented at bauMax. Improved computing power and data handling facilities at continuously decreasing cost, advanced research techniques for processing hundreds of sales response functions simultaneously, and modern point-of-sale technology such as electronic shelf labeling systems, should enable companies like bauMax to reduce the menu costs of dynamic retail pricing systems in the very near future.

However, putting aside all these technological aspects, one important lesson can be learned from the bauMax success story. If marketing intelligence systems are implemented imaginatively, they do live up to the claims made for them. The lesson we can learn from this project is that getting skeptical managers and users onside in the early stages of implementation is crucial. If such issues can be adequately resolved, automated pricing systems face a bright future. 